\title{
Seroprevalence of SARS coronavirus antibody in household contacts
}

\author{
C.-C. LEE ${ }^{1}$, S.-Y. CHEN ${ }^{1}$, I.-J. CHANG ${ }^{2}$, P.-C. TSAI ${ }^{1}$, T.-C. LU ${ }^{1}$, P.-L. W U ${ }^{1}$, \\ W.-J. CHEN ${ }^{1}$, L.-M. HUANG ${ }^{3}$ AND S.-C. CHANG ${ }^{4 *}$ \\ ${ }^{1}$ Department of Emergency Medicine, National Taiwan University Hospital, Taipei, Taiwan \\ ${ }^{2}$ Institute of Preventive Medicine, School of Public Health, National Taiwan University, Taipei, Taiwan \\ ${ }^{3}$ Division of Infectious Disease, Department of Pediatrics, National Taiwan University Hospital, Taipei, Taiwan \\ ${ }^{4}$ Division of Infectious Disease, Department of Internal Medicine, National Taiwan University Hospital, \\ Taipei, Taiwan
}

(Accepted 21 March 2005, first published online 8 July 2005)

\section{SUMMARY}

Between March and July 2003, 671 cases of severe acute respiratory syndrome (SARS) were diagnosed in Taiwan with a total of 84 fatalities. After the epidemic, a serological survey was conducted involving the asymptomatic household contacts. Household contacts of 13 index patients were enrolled in the study. Contact history and clinical symptoms of the household contacts were recorded by standardized questionnaires. Blood samples of patients and household contacts were collected at least 28 days after symptom onset in the index patients or household exposure in the contacts for SARS-associated coronavirus (SARS-CoV) IgG testing. On the basis of this investigation, 29 persons ( 25 adults and 4 children) were identified as having had unprotected exposure to the index cases before infection-control practices were implemented. Laboratory evaluation of clinical specimens showed no evidence of transmission of SARS-CoV infection to any contacts. This investigation demonstrated that subclinical transmission among household contacts was low in the described setting.

\section{INTRODUCTION}

Severe acute respiratory syndrome (SARS) is an emerging infectious disease caused by a novel SARSassociated coronavirus (SARS-CoV) [1]. It has caused outbreaks in China, Hong Kong, Taiwan, Singapore and Canada and 8096 cases of probable SARS were reported globally in 2003 [2, 3]. The case-fatality rate was $13 \%$ for patients $<60$ years of age and $43 \%$ for those aged $\geqslant 60$ years [4]. SARS-CoV is highly infectious in the hospital setting. Health-care workers

\footnotetext{
* Author for correspondence: S.-C. Chang, M.D., Ph.D, Department of Internal Medicine, National Taiwan University Hospital, No. 7 Rd, Chung-Shan South, Taipei, Taiwan 100.

(Email: sc4030@ha.mc.ntu.edu.tw)
}

accounted for $37-63 \%$ of suspected SARS cases in some case series [4-7]. On the other hand, household transmission seemed to be less efficient. A significant number of household close contacts remained asymptomatic after unprotected exposure. Whether subclinical infections occur among family members and whether they may seroconvert to the SARS-CoV with minimal or no symptoms remains unknown. Therefore, a serology survey was conducted on these household contacts at least 28 days after unprotected exposure to the symptomatic index patients. We wanted to elucidate factors influencing household transmission of SARS-CoV and predict possible population immunity against SARS-CoV after the epidemic. 


\section{METHODS}

\section{Study design and population}

The study was a cross-sectional survey. We invited 63 consecutive SARS patients to join the study. Initial treatment was carried out in the Emergency Department of the National Taiwan University Hospital from March to May 2003. The modified World Health Organization (WHO) criteria was used as the case definition, including temperature of $\geqslant 38{ }^{\circ} \mathrm{C}$, cough or shortness of breath and new pulmonary infiltrates shown on chest X-ray in the absence of an alternative diagnosis to explain the clinical signs and symptoms [8, 9]. All patients' infections were either confirmed serologically or SARSCoV RNA was detected by RT-PCR.

A household was defined as a residential place with a unique address. A household index was a person with probable SARS and the first person to introduce SARS into the household. A household close contact was defined as a person living in the same household as the household index during their illness and having cared for, lived with, or had direct contact with respiratory secretions or body fluids of a suspect or probable case of SARS.

\section{Survey content and administration}

\section{Data collection}

All probable SARS case-patients were informed by a letter briefly describing the nature of the study. Blood of the participants was collected for SARS-CoV IgG analysis. Contact history was recorded by a standardized questionnaire with interviews conducted by physicians. The questionnaire collected basic demographics, exposure duration from fever onset to hospitalization and behaviour regarding close contact with the index patient. The study was approved by the hospital Ethics Committee and written informed consent was provided by all participants.

\section{Data analysis}

\section{Laboratory examination}

SARS-CoV IgG was detected by a standard indirect immunofluorescence antibody assay (IFA). Spot slides for IFA were prepared by applying the suspension mixed with SARS-CoV-infected Vero E6 cells and uninfected cells. Slides were dried and fixed in acetone. The conjugates used were goat antihuman $\mathrm{IgG}$ conjugated to fluorescein isothiocyanate (Organon Teknika-Cappel, Turnhout, Belgium). The starting dilution for serum specimens was $1: 25$. The assay sensitivity has been shown to be $99 \cdot 1 \%$, with a specificity, positive predictive value and negative predictive value of $87 \cdot 8,88 \cdot 1$ and $99 \cdot 1 \%$ respectively [10].

\section{Statistical analysis}

Fisher's exact test, $\chi^{2}$ test, or Student's $t$ test was used where appropriate. A $P$ value of $<0.05$ was considered statistically significant. Statistical analysis was performed using SPSS software, version 12.0 (SPSS Inc., Chicago, IL, USA).

\section{RESULTS}

The 63 patients with probable SARS resided in 59 households. Of these, four patients lived in the same households as the index cases and were considered as co-primary cases because they had similar exposure and similar time of illness onset. A total of 29 household contacts from 13 households completed the questionnaires and had serum samples collected. Contacts from the remaining $46(79.03 \%)$ households were not included because two did not have household members and the rest declined participation. The basic demographics, severity of disease on presentation and exposure duration from fever onset to hospitalization between participating and nonparticipating patients were not statistically different (see Table). The participating index patients were distributed mainly in the middle course of the epidemic, with one in March, nine in April and three in May. None were health-care workers. Of the participating contacts, the mean age was $37 \cdot 8$ years [range 3-89, $95 \%$ confidence interval (CI) 33.82-41.73], with four paediatric contacts aged $<15$ years of age (mean $10 \cdot 2$, $95 \%$ CI $5 \cdot 32-15 \cdot 13)$. The mean length of exposure was $4 \cdot 2$ days (range $2-7,95 \%$ CI $2 \cdot 40-6 \cdot 06$ ). Sharing the same toilet $(86 \cdot 2 \%, 25 / 29)$ was the most frequent contact behaviour, followed by sharing the same dish of food $(75.9 \%, 22 / 29)$, hugging $(31 \cdot 0 \%, 9 / 29)$ and sleeping together $(31 \cdot 0 \%, 9 / 29)$. Twenty-seven out of $29(93 \cdot 1 \%)$ household contacts did not have any noticeable illness after exposure. Of the two household contacts reporting symptoms during the period, one had self-limited watery diarrhoea and the other had a chronic cough. The interval between household exposure and blood sampling ranged from 78 to 121 days, with a mean of 93.8 days $(95 \%$ CI 
Table. Demographic and clinical features between the participating and non-participating index patients

\begin{tabular}{llll}
\hline \hline & $\begin{array}{l}\text { Participating } \\
\text { index patients }\end{array}$ & $\begin{array}{l}\text { Non-participating } \\
\text { index patients }\end{array}$ & $P$ value \\
\hline Patient $(n)$ & 13 & 50 & $0 \cdot 537$ \\
Age, mean \pm s.D. (years) & $41 \cdot 15 \pm 14 \cdot 50$ & $44 \cdot 10 \pm 15 \cdot 41$ & $0 \cdot 719$ \\
Male-to-female ratio, $\%$ & $38 \cdot 5$ & $44 \cdot 0$ & $0 \cdot 668$ \\
Mortality, $n(\%)$ & $1(7 \cdot 7)$ & $8(18 \cdot 2)$ & $0 \cdot 27$ \\
Abnormal chest radiography & $8(61 \cdot 5)$ & $42(80 \cdot 0)$ & $0 \cdot 266$ \\
on presentation, $n$ (\%) & & $850 \cdot 95 \pm 310 \cdot 45$ & \\
Lymphocyte count on & $970 \cdot 56 \pm 450 \cdot 24$ & & $0 \cdot 834$ \\
presentation, mean \pm s.D. & & $4 \cdot 38 \pm 2 \cdot 37$ & \\
$\quad$ per mm ${ }^{3}$ ) & $4 \cdot 23 \pm 1 \cdot 83$ & & \\
Fever duration before & & & \\
hospitalization, mean \pm s.D. (day) & & & \\
\hline \hline
\end{tabular}

S.D., Standard deviation.

83.64-103.97). None of the 29 household contacts had SARS-CoV IgG. All $12(100 \%)$ serum samples from index patients in the convalescent phase were positive for SARS-CoV IgG. One index patient died before the study started and a diagnosis of SARS was confirmed by RT-PCR.

\section{DISCUSSION}

SARS-CoV transmission is now understood to involve close contact with symptomatic patients [1-3]. Previous research focused mainly on the risk of secondary transmission among household contacts, while our investigation addressed the seroepidemiology and clinical manifestations of the non-case household close contacts [11, 12]. As shown in this investigation, none of the household contacts seroconverted to SARS-CoV.

There were some limitations to this study. First, transmission in the households might not have been as efficient compared with the hospital setting, as shown by reports from Canada, Philippines and United States where the household transmission rate was $<6 \%$ [13-15]. One explanation for this might be that household exposure usually occurred in the early stages of the disease while transmission efficiency appears to be greatest during the second week of illness [16]. Data from Singapore showed that few secondary cases occurred when symptomatic cases were isolated within 5 days of illness onset [17]. Similarly, RT-PCR data from Hong Kong indicate that viral excretion in nasopharyngeal aspirate specimens peaked on days 12-14 of illness [18]. All household exposure in our study occurred within 1 week of symptom onset. Hence, the chance of being exposed to respiratory secretions with high viral loads was greatly reduced.

The risk of exposure to faeces of the index patients could not be ignored in this investigation. Twenty-five out of 29 contacts $(86 \cdot 2 \%)$ reported having shared toilet facilities with the index patient. Previous studies reported that viral excretion in faeces was two orders of magnitude greater than in nasopharyngeal aspirate specimens. They also reported that SARS-CoV RNA detection in faeces peaked between 9 and 14 days [18]. Although the absence of seroconversion could also be explained by the relatively low viral shedding in faeces in the early course of illness, it was also possible that SARS-CoV might exist in a non-transmissible form in faeces. Previous reports have shown that no viable virus could be grown from faecal specimens despite substantial viral shedding detected in these samples [12].

We tried to calculate the sample size required to determine a transmission rate with a $95 \% \mathrm{CI}$ of $\pm 10 \%$ and a two-tailed significance level of 0.05 . According to previous reports, the transmission rate among the household contacts would be lower than that among the hospital contacts. In a Canadian case series, nursing staff having contact with probable SARS case-patients in the emergency department had a transmission rate of $44 \%$ [6]. If we assumed the risk of transmission was equal in all participating contacts and probably half of that in the Canadian case series $(22 \%)$, at least 65 participants should be included in the study. Our study has a reduced sample size of 29 patients, which would result in a wider confidence interval of $\pm 15 \%$. When determining whether the 
seropositive rate in our study was equal to that among the general population, the study was also underpowered. A recent study involving 938 healthy Hong Kong adults, whose serum had been stored as part of a hepatitis B serosurvey in 2001, indicated that SARSCoV IgG was detected in $1.8 \%$ of the samples [19]. In comparison with the results, our study has a $0 \cdot 1 \%$ calculated power to detect a relative $50 \%$ difference from that sample.

Results of this investigation should be interpreted in light of these limitations. The small number of participants does not allow for accurate estimation of the risk of transmission to household members. Moreover, the survey is vulnerable to both recall and reporting bias. Finally, contacts who refused to participate might have done so because they were concerned about the risk or suffered from the psychological stress of exposure to the hospital and health-care personnel, since the study was carried out 1.5 months after the SARS epidemic in Taiwan.

In conclusion, this study characterized the seroepidemiology of the asymptomatic household close contacts. We have shown that the risk of subclinical transmission among household contacts was low in the described setting and immunity to SARS-CoV among household contacts may be low. Given that the study numbers were limited, a larger scale analysis of the asymptomatic close contacts in past SARS cases would be helpful in verifying these findings.

\section{REFERENCES}

1. Ksiazek TG, Erdman D, Goldsmith CS, et al. A novel coronavirus associated with severe acute respiratory syndrome. N Engl J Med 2003; 348: 1953-1966.

2. World Health Organization. Outbreak news - severe acute respiratory syndrome (SARS). Wkly Epidemiol Rec 2003; 78: 81 .

3. World Health Organization. Summary of probable SARS cases with onset of illness from 1 November 2002 to 31 July 2003 (http://www.who.int/csr/sars/country/ table2004_04_21/en/). Accessed 16 March 2005.

4. Donnelly $\overline{\mathbf{C A}}$, Ghani AC, Leung GM, et al. Epidemiological determinants of spread of causal agent of severe acute respiratory syndrome in Hong Kong. Lancet $2003 ; 361$ : 1761-1766.
5. Twu SJ, Chen TJ, Chen CJ, et al. Control measures for severe acute respiratory syndrome (SARS) in Taiwan. Emerg Infect Dis 2003; 9: 718-720.

6. Varia M, Wilson S, Sarwal S, et al. Investigation of a nosocomial outbreak of severe acute respiratory syndrome (SARS) in Toronto, Canada. Can Med Assoc J 2003; 169: 285-292.

7. Masur H, Emanuel E, Lane HC. Severe acute respiratory syndrome: providing care in the face of uncertainty. J Am Med Assoc 2003; 289: 2861-2863.

8. World Health Organization. Case definitions for surveillance of severe acute respiratory syndrome (http:// www.who.int/csr/sars/archive/en/). Accessed 20 May 2004.

9. Peiris JSM, Chu CM, Cheng VCC, et al. Clinical progression and viral load in a community outbreak of coronavirus-associated SARS pneumonia: a prospective study. Lancet $2003 ; 361$ : 1767-1772.

10. Wu HS, Chiu SC, Tseng TC, et al. Serologic and molecular biologic methods for SARS-associated coronavirus infection, Taiwan. Emerg Infect Dis 2004; 10: 304-310.

11. Goh DL-M, Lee BW, Chia KS, et al. Secondary household transmission of SARS, Singapore. Emerg Infect Dis $2004 ; \mathbf{1 0}: 232-234$.

12. Isakbaeva ET, Khetsuriani N, Beard RS, et al. SARSassociated coronavirus transmission, United States. Emerg Infect Dis 2004; 10: 225-231.

13. Avendano M, Derkach P, Swan S. Clinical course and management of SARS in health care workers in Toronto: a case series Can Med Assoc J 2003; 168: 1649-1660.

14. Anon. Sars Outbreak in the Philippines. Wkly Epidemiol Rec 2003; 78: 189-196.

15. Seto WH, Tsang D, Yung RW, et al. Effectiveness of precautions against droplets and contact in prevention of nosocomial transmission of severe acute respiratory syndrome (SARS). Lancet $2003 ; 361$ : 1519-1520.

16. World Health Organization. Consensus document on the epidemiology of severe acute respiratory syndrome (SARS) (http://www.who.int/csr/sars/en/WHOconsen sus.pdf). Accessed 16 Jan. 2005.

17. Gay N, Ma S. Presentation on the modeling of data from Singapore, Global Meeting on the Epidemiology of SARS World Health Organization, Geneva, Switzerland, 16-17 May 2003.

18. Cheng PK, Wong DA, Tong LK, et al. Viral shedding patterns of coronavirus in patients with probable severe acute respiratory syndrome. Lancet 2004; 363: 1699-1700.

19. Zheng BJ, Wong KH, Zhou J, et al. SARS-related virus predating SARS outbreak, Hong Kong. Emerg Infect Dis $2004 ; 10: 176-178$. 